\title{
SERUM LDL CHOLESTEROL/HDL CHOLESTEROL RATIO ALTERNATIVE TO ALBUMIN IN RELATION TO CORONARY HEART DISEASE
}

\author{
S. PARVEEN ${ }^{1}$, MD.SHAMSUZZAMAN ${ }^{2}$, KHURSID ARA BEGUM ${ }^{3}$, DR.NIZAMUL HAQUE BHUIYAN ${ }^{4}$, \\ R.YEASMIN ${ }^{5}$, SAIFUNNAHAR ${ }^{6}$
}

\begin{abstract}
:
In a study of coronary heart disease in males, their is a correlation between LDL cholesterol/ HDL cholesterol ratio \& albumin $(r=0.46, p<0.001)$. We then correlated the LDL cholesterol: albumin ratio( TC: Alb) with the LDL $c: H D L-c$ ratio $(r=0.12, p<0.001)$. An excellent correlation was obtained between $L D L-C:$ Alb ratio separated the patients with normal $(<5)$ and increased (>5) LDL-C: HDL-C ratio better than LDL-C by itself(Amin A Nanji, Suseela Reddy et al).
\end{abstract}

\section{Introduction:}

A wide variety of epidemiological data indicates that individual lipoproteins are better predictor of risk factor for coronary heart disease than are the levels of total plasma lipid alone (Castelli WP, Kannel WB et al). Fasting LDL-C level, albumin \& high density lipoprotein levels were obtained from 45 men aged between 45 to 65 yrs at Dhaka medical college hospital. At this older ages the major potent lipid risk factor was HDL $-\mathrm{C}$, which had an inverse association with the incidence of coronary artery disease $(<0.001)$ in men. These lipids were associated with each major manifestation of coronary heart disease ${ }^{2}$.

Lipids and lipoproteins studies in the past have generally emphasized the positive relationship of total cholesterol, low density lipoprotein, very low density lipoprotein\& triglyceride to the risk of coronary heart disease. It appeared that essentially all of the cholesterol \& phospholipids of normal plasma is combined with protein \& presumbly in combination either with alpha lipoproteins or with beta lipoproteins. The high density lipoprotein (HDL) or lipoprotein appeared to have an inverse relation to the risk of coronary heart disease, the lower their concentration the greater the risk of coronary heart diseases. This anomalous relationship where high levels appear to be protective may be one reason that HDL has received little attention from investigators. We attempted to determine whether the total LDL-C: Albumin (LDL-C: Alb) ratio, correlated with the LDL-C: HDL-C ratio could be useful clinically. An excellent correlation was obtained between LDL-C: Alb and LDL-c: HDL-C $(\mathrm{r}=0.12, \mathrm{p}<0.001)$ using the LDL-C: HDL-C as and index of cardiovascular risk. HDL-c may be important for the normal clearance of cholesterol from tissues. Although cholesterol is synthesized in most tissues (Dya A J, Wilkilson G K, et al), it can be catabolized \& quantified only by the liver( Nestel $\mathrm{P}$ et al) ${ }^{3}$. Cholesterol must be transported from these tissues to the liver during cholesterol turnover. Cholesterol exists between plasma lipoprotein \& tissues. The activity of the plasma cholesterol esterifying enzyme, lecithin-cholesterol acryl transferase ( LCAT) promote the transfer of cholesterol from erythrocytes to plasma by maintaining the body in the esterified \& unspecified forms, but latter a shift of the equilibrium between plasma cell membrane unesterified cholesterol (Murfy J et al) ${ }^{4}$. It is important to identify these are infact protective against atherosclerosis, and their hypercholesterolemia should not be treated. Identification of these individual requires determination of HDL-C. Because HDL-C measurements are expensive, a cheaper alternative would considerably reduce medical cost. In a study of apparently healthy males, we noted a correlation between serum albumin and HDL-C concentration $(\mathrm{r}=0.32, \mathrm{p}<0.001)$. Because of this observation we attempted to determine whether the LDL-C: Albumin ratio (a) correlated with LDL-C: HDL-C and (b) could be useful clinically.

\section{Patients \& method:}

Blood samples were obtained from 50 men (age range 45-65 yr) referred to the coronary care unit at Dhaka

1. Associate Professor, Dept. of Biochemistry, Ibrahim Medical College.

2. Associate Professor, Dept. of Orthopedics, Dhaka Medical College.

3. Associate Professor, Dept. of Biochemistry, Bangladesh Medical College

4. Associate Professor, Dept. of Institute of Food \& Nutrition, Dhaka University.

5. Assistant Professor, Dept. of Biochemistry, Ibrahim Medical College.

6. Associate Professor, Dept. of Anatomy, Holy Family Red Crescent Medical College. 
medical college hospital. All were coronary heart disease patient. Blood for analysis was drawn by venepancture from the subject after they had fasted for 12 to 14 hour overnight. Serum LDL $-\mathrm{C} \&$ albumin were measured by an automated multiple analysis system. HDL - C was measured after precipitation of lipoprotein containing Apo-B with heparin \& manganese chloride. The range of values in the subjects for parameter studied were LDL$\mathrm{C}<135 \mathrm{mg} \%$, HDL-C $25-55 \mathrm{mg} / \mathrm{dl}$, and albumin 3.2-7 $\mathrm{g} / \mathrm{dl}$. Since measurement of TC \& albumin is routinely available in multichannel analyzers, use of the LDL: Alb ratio could considerably reduce the number of HDL-C measurements necessary.

Statistical analysis: For statistical analysis student " $\mathrm{t}$ " test. Pearson's correlation coefficient test ( $\mathrm{t}$ ) was used as the test of significance. $P$ value of $<0.05$ was considered as the level of significance.

\section{Results:}

\section{Descriptive statistics}

\begin{tabular}{lccccc}
\hline & $\mathrm{N}$ & Minimum & Maximum & Mean & SD \\
\hline AGE & 37 & 40 & 67 & 59.38 & 6.18 \\
LDL-C & 45 & 138 & 220 & 180.69 & 19.48 \\
HDL-C & 44 & 25 & 55 & 40.25 & 8.25 \\
Alb & 45 & 3.20 & 7.00 & 4.5422 & 0.7047 \\
\hline
\end{tabular}

Table 1: All results are show as their mean values \& $\mathrm{SD}$ (standard deviation) in $\mathrm{mg} / \mathrm{dl}$

LDL-C level mean 180.69(SD-19.48), HDL level mean 40.25(SD-8.25), Alb level mean 4.5422(SD-0.7047)

Comparison between LDL-chol. / Alb. and HDL Ratio

\begin{tabular}{lccccc}
\hline & Mean & SD & SEM & t-test & $\mathrm{p}$ \\
\hline Age & 60.36 & 4.87 & 0.85 & 3.104 & 0.004 \\
& 51.25 & 10.31 & 5.15 & & \\
LDL-C & 177.54 & 17.77 & 2.92 & -1.066 & 0.293 \\
& 188 & 26.88 & 13.44 & & \\
HDL & 41.28 & 8.30 & 1.38 & 1.082 & 0.286 \\
& 36.50 & 9.18 & 4.59 & & \\
Alb. & 4.5297 & 0.7593 & 0.1248 & -0.052 & 0.959 \\
\hline
\end{tabular}

We tested the association between Alb. /LDL-C ratio and HDL. There was no significant correlation between the two variables in the study population.

\section{Pearson's correlation among variables}

\begin{tabular}{lcccccc}
\hline & & Age & Chol & HDL & Alb. & TG \\
\hline Age & P. Correlation & 1.000 & -0.077 & 0.132 & 0.080 & 0.826 \\
& Sig (2-tailed) & & 0.653 & 0.444 & 0.637 & 0.381 \\
& $\mathrm{~N}$ & 37 & 37 & 36 & 37 & 3 \\
LDL-C & P. Correlation & -0.077 & 1.000 & 0.461 & 0.124 & 0.243 \\
& Sig (2-tailed) & 0.653 & & 0.002 & 0.415 & 0.694 \\
& $\mathrm{~N}$ & 37 & 45 & 44 & 45 & 5 \\
HDL & P. Correlation & 0.132 & 0.461 & 1.000 & 0.262 & 0.949 \\
& Sig (2-tailed) & 0.444 & 0.002 & & 0.086 & 0.051 \\
& $\mathrm{~N}$ & 36 & 44 & 44 & 44 & 4 \\
Alb. & P. Correlation & 0.080 & 0.124 & 0.262 & 1.000 & -0.825 \\
& Sig (2-tailed) & 0.637 & 0.415 & 0.086 & & 0.086 \\
& $\mathrm{~N}$ & 37 & 45 & 44 & 45 & 5 \\
\hline
\end{tabular}

LDL-C: Alb ratio-0.1415 P=0.39(r=0.12 $\mathrm{p}=0.42)$

LDL: HDL Significant positive correlation, $(\mathrm{r}=0.46 \mathrm{p}<0.01)$

P. correlation-Pearson's correlation.

There was significant positive association between LDL-C\& HDL $(r=0.46 \mathrm{p}=0.002)$ 


\section{Discussion:}

We are not aware of the correlation between serum albumin and HDL-C and serum albumin concentrations have never been shown to have a predictive value for coronary disease.

Based on this relation between HDL-C and albumin, LDL-C: Albumin ratio was used to discriminate between subjects with normal \& increased LDL-C: HDL-C. The LDL-C: Albumin ratio performed better than LDL-C by itself (Gordon T, Castelli WP et al). However it is important to note that the high correlation between LDL-C: Alb and LDL-C: HDLC. The LDL-C: HDL-C ratio is most helpful in distinguishing patients with a modest hypercholesterolemia due to increased HDL from patients whose hypercholesterolemia is due to increased light density lipoprotein (Amin A Nanji, Suseela Reddy) ${ }^{5}$.

These data provide evidence of a strong negative association of HDL $-\mathrm{C}$ level with the subsequent incidence of coronary heart disease in men over age $50 .$. The for going association raise the possibility that low plasma concentrations of HDL may be involved in the pathogenesis of coronary atherosclerosis

The use of LDL-C: Alb ratio would help in considerably reducing the number of HDL-C determination necessary. Both LDL-C \& Albumin are routinely available on multichannel analysis.

Gordon T, Castelli WP showed that the ratio is useful only in those subjects with modest hypercholesterolemia since individual with very high LDL-XC concentration are unlikely to have their risk of developing CHD altered either by HDL-
Cholesterol measurements. Since LACT is implicated in the production of HDL cholesterol esters, increased amount of albumin may be necessary to remove an increased amount of lysolecithin formed as a result of increased LDL -C \& HDL -Cholesterol ester formation ${ }^{1-3}$.

In accordance with Kannel WP, Tall AR et al with this hypothesis, it is further proposed that the development of atherosclerosis might be more successful prevented by the increasing plasma HDL $\&$ hence the clearance of cholesterol from the arterial wall, than by conventional attempts to reduce the plasma cholesterol \& other lipoproteins alone in our study $^{2,4}$.

We can say that LDL-C: Alb is a better predictor for CHD than LDL-C: HDL-C.

\section{References:}

1. Amin A nanji, Suseela Reddy. Use of total cholesterol / Albumin ratio as an alternative to highdensity lipoprotein cholesterol measurement. J Clin Pathol 1983; 36: 112-7.

2. Kannel WB, Castelli WP, Gordon T. Cholesterol in the predictor of atherosclerosis disease. Ann Intern Med 1987; 90: 21-5.

3. Gordon T, Castelli WP, H Jortland MC, Dawbwer TR, High density lipoprotein as a protective factor against coronary heart disease . Am J Med 1997; 62: $6-13$.

4. Tall AR, Small DM. Plasma high density lipoprotein s. N Engl J Med 1978; 299: 210-17.

5. G. J. Miller, N. E. Miller. Plasma high density lipoprotein concentration and development ischemia heart disease. The Lancet 1995; 5: 113-7 